\title{
The lithium content of the globular cluster NGC $6397^{\star}$
}

\author{
P. Bonifacio ${ }^{1}$, L. Pasquini ${ }^{2}$, F. Spite ${ }^{3}$, A. Bragaglia ${ }^{4}$, E. Carretta ${ }^{5}$, V. Castellani ${ }^{6}$, M. Centuriòn ${ }^{1}$, A. Chieffi ${ }^{7}$,
} R. Claudi ${ }^{5}$, G. Clementini ${ }^{4}$, F. D’Antona ${ }^{8}$, S. Desidera ${ }^{5,9}$, P. François ${ }^{3}$, R. G. Gratton ${ }^{5}$, F. Grundahl ${ }^{10}$, G. James ${ }^{3}$, S. Lucatello ${ }^{5,9}$, C. Sneden ${ }^{11}$, and O. Straniero ${ }^{12}$

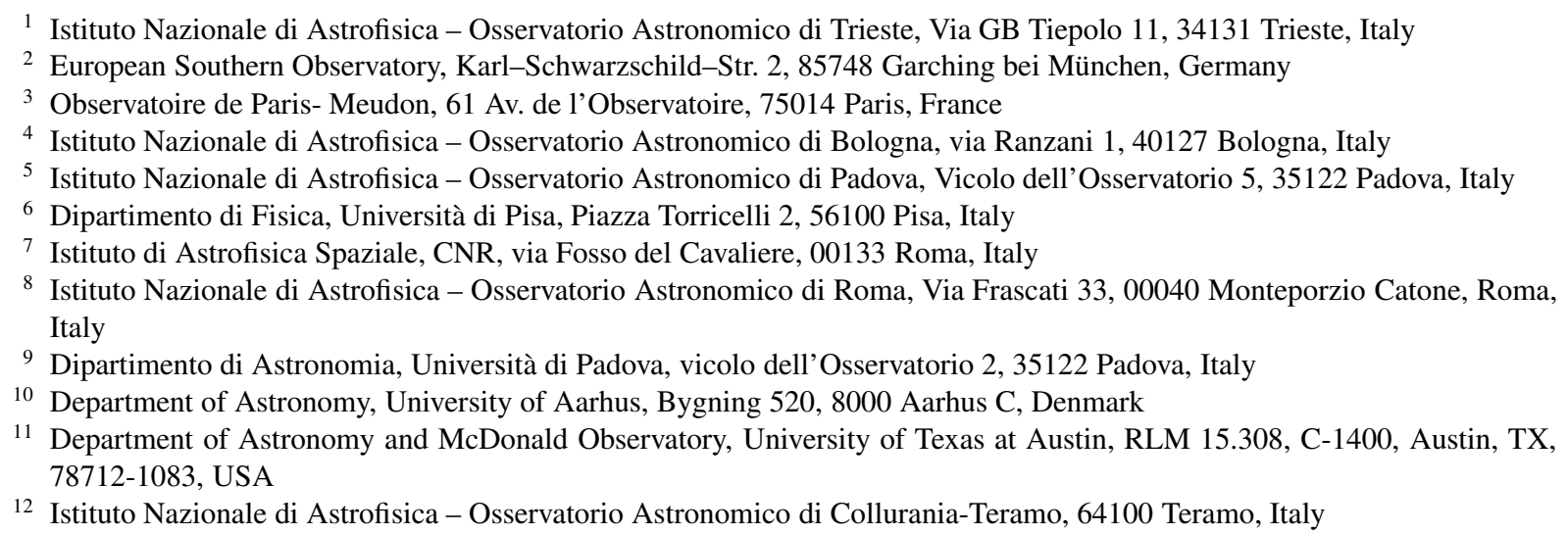

Received 1 March 2002 / Accepted 15 April 2002

\begin{abstract}
We make use of high resolution, high signal-to-noise ratio spectra of 12 turn-off stars in the metal-poor globular cluster NGC 6397 to measure its lithium content. We conclude that they all have the same lithium abundance $A(\mathrm{Li})=2.34$ with a standard deviation of $0.056 \mathrm{dex}$. We use this result, together with Monte Carlo simulations, to estimate that the maximum allowed intrinsic scatter is of the order of 0.035 dex. This is a new stringent constraint to be fulfilled by stellar models which predict Li depletion. We argue that although a mild depletion of $0.1-0.2$ dex, as predicted by recent models, cannot be ruled out, there is no compelling reason for it. This fact, together with the good agreement with the Li abundance observed in field stars, supports the primordial origin of lithium in metal-poor stars. Taking the above value as the primordial lithium abundance implies a cosmic baryonic density which is either $\Omega_{\mathrm{b}} h^{2}=0.016 \pm 0.004$ or $\Omega_{\mathrm{b}} h^{2}=0.005_{-0.0006}^{+0.0026}$, from the predictions of standard big bang nucleosynthesis. The high baryonic density solution is in agreement with recent results on the primordial abundance of deuterium and ${ }^{3} \mathrm{He}$ and on the estimates derived from the fluctuations of the cosmic microwave background.
\end{abstract}

Key words. diffusion - stars: abundances - stars: atmospheres - stars: Population II - Galaxy: globular clusters: NGC 6397 cosmology: observations

\section{Introduction}

The lithium abundance in metal-poor turn-off stars appears as a plateau, independent of metallicity and effective temperature (Spite \& Spite 1982a; Spite \& Spite 1982b), the so-called Spite plateau. Currently its most widely accepted interpretation is that the lithium observed in metal-poor stars has been produced in the big bang and is thus a probe of the universal baryonic density. Recent claims that the Spite plateau does not reflect the true primordial abundance which would be either lower, due to

Send offprint requests to: $\mathrm{P}$. Bonifacio,

e-mail: bonifaci@ts.astro.it

* Based on observations made with the ESO VLT-Kueyen telescope at the Paranal Observatory, Chile, in the course of the ESO-Large program 165.L-0263. chemical evolution effects (Ryan et al. 1999), or higher due to Li depletion (Pinsonneault et al. 1999, 2002; Salaris \& Weiss 2001; Théado \& Vauclair 2001), deal with second order effects. The primordial $\mathrm{Li}$ abundance is within $\sim 0.2$ dex of the plateau value. Thus there is a general consensus that the bulk of lithium in metal-poor stars is of primordial origin. If this is the case we expect metal-poor stars, wherever in the universe, to show the same Li abundance. The present instrumentation is not capable of providing Li abundances of turn-off stars in nearby external galaxies, however Molaro (1997) suggested that extra-galactic Li has already been detected, at the plateau value, in the bluemetal-poor star CS 22873-139, possibly accreted from an external galaxy. In spite of this, our present knowledge rests on the study of $\mathrm{Li}$ abundances in different metal-poor populations of our own Galaxy. The most studied population is the metal-poor 
field halo (Spite \& Spite 1982b; Bonifacio \& Molaro 1997; Ryan et al. 1999, and references therein). Molaro et al. (1997) have studied lithium in metal-poor thick disc candidates and found that they lie on the Spite plateau; two more thick disc stars lying on the plateau were noted by Romano et al. (1999).

Molaro \& Pasquini (1994) and Pasquini \& Molaro (1996) were the first to study Li in turn-off stars in NGC 6397 and found that it is consistent with the plateau. Recently Thévenin et al. (2001) published Li abundances for seven turn-off stars of NGC 6397, based on UVES data similar to our own, confirming the earlier results. Boesgaard and collaborators have studied lithium in the globular clusters M 92 (Boesgaard et al. 1998), M 13 and M 71 (Boesgaard et al. 2000) using the $10 \mathrm{~m}$ Keck telescope and found a sizeable scatter in $\mathrm{Li}$ abundances in all three clusters.

The globular cluster NGC 6397 is one of the nearest and best studied ones. It is one of the main targets of the ESOLarge Program 165.L-0263 which we are conducting with the aim of providing accurate abundances, reddenings and distances for globular clusters with a range of metallicities. In Gratton et al. (2001) we provided the abundances of several elements and reached the conclusion that NGC 6397 is a very homogeneous cluster, from the chemical point of view, down to the main-sequence stars. It is also quite metal-poor $([\mathrm{Fe} / \mathrm{H}]=$ $-2.03 \pm 0.02$ ). We deferred the study of $\mathrm{Li}$ abundances in turnoff stars to the present paper because we believe lithium deserves special care. From an observational point of view the Li I $670.8 \mathrm{~nm}$ resonant doublet, which is the only lithium line observed, is rather weak, asymmetric and difficult to measure accurately in faint objects. From the point of view of the analysis $\mathrm{Li}$ is extremely sensible to the adopted effective temperature, since it is mostly ionized ( $\mathrm{Li} \mathrm{II}>99.7 \%$ ) in the atmospheres of solar-type stars and neutral lithium is only a trace species. Combining the data from Thévenin et al. (2001) with our own, the total number of turn-off stars of NGC 6397 observed at high resolution with a high $S / N$ ratio is twelve. Such a large sample allows us to tackle the issue of the possible intrinsic dispersion of the Li content among turn-off stars in this cluster.

\section{Observations}

Our spectra were collected at ESO-Paranal with the UVES spectrograph (Dekker et al. 2000) at the Kueyen $8.2 \mathrm{~m}$ telescope between June 15th 2000 and June 22nd 2000, and have already been described in Gratton et al. (2001); the log of the observations is given in Table 1, the DIMM seeing was noted, when available. While in Gratton et al. (2001) we have relied on the pipeline, in this paper we reduced all the spectra beginning from the raw data using the MIDAS echelle context. The purpose was both to check the accuracy of the pipeline reduction and to try to improve it, especially trying to get rid of the low frequency variations in the continuum, at the level of a few per-cent which were apparent in the pipeline data.

The stability of the UVES spectrograph proved to be remarkable: the flat-fields were perfectly reproducible from one night to the other, except for the night of the 22nd, for which a slight shift of the order positions of a couple of pixels

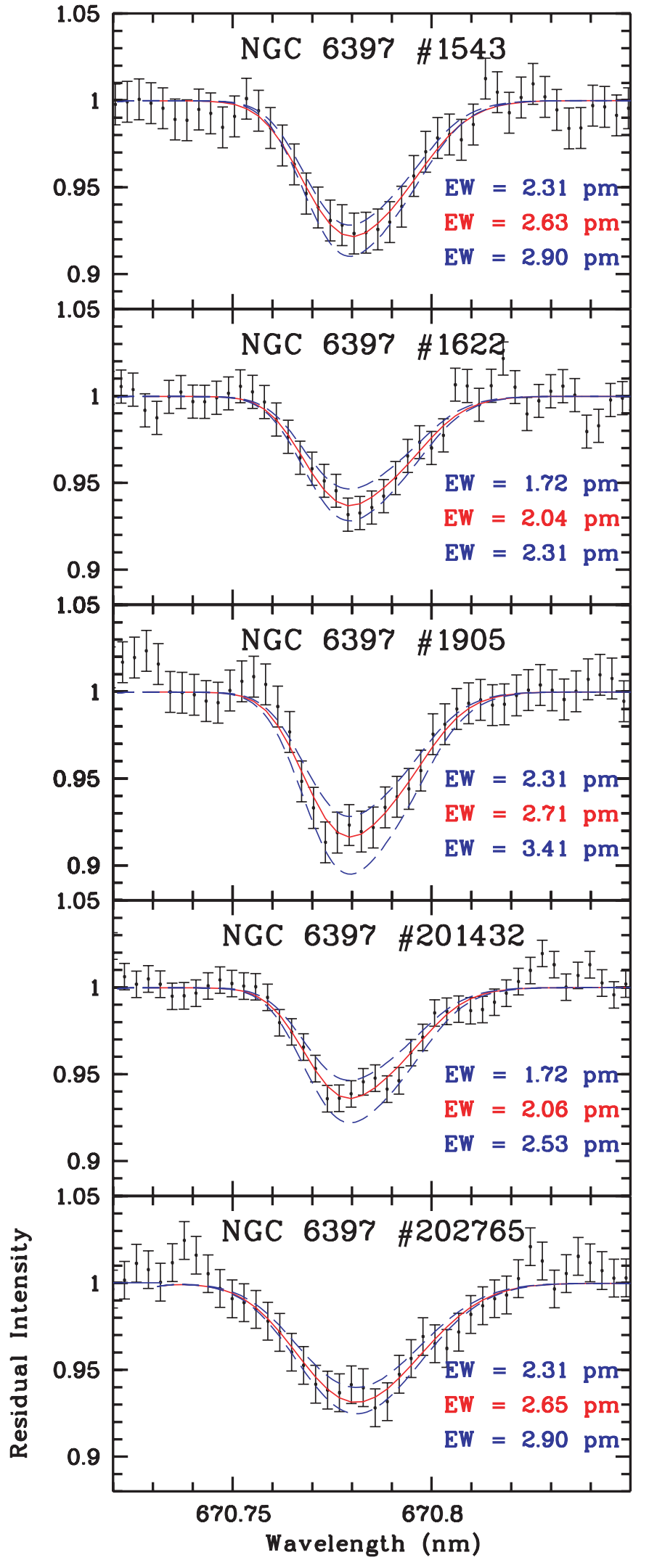

Fig. 1. Li doublet for the program stars.

appeared; the wavelength scale was also extremely stable, the largest peak-to-peak variation in the position of the arc lines observed on different nights was $0.005 \mathrm{~nm}$. The $F W H M$ of the 
Table 1. Log of the observations.

\begin{tabular}{lcccl}
\hline \hline star \# & $\begin{array}{c}\text { date } \\
\text { d/m/y }\end{array}$ & $\begin{array}{c}\text { UT } \\
\text { h:m:s }\end{array}$ & $\begin{array}{c}t_{\text {exp }} \\
\text { s }\end{array}$ & $\begin{array}{l}\text { seeing } \\
\text { arcsec }\end{array}$ \\
\hline 1543 & $20 / 06 / 2000$ & $00: 56: 06$ & 4500 & 0.8 \\
1543 & $20 / 06 / 2000$ & $02: 12: 44$ & 4500 & 0.8 \\
1543 & $20 / 06 / 2000$ & $03: 30: 21$ & 3600 & 1.0 \\
1543 & $20 / 06 / 2000$ & $04: 33: 03$ & 4500 & 1.1 \\
1622 & $16 / 06 / 2000$ & $02: 21: 00$ & 3600 & 0.6 \\
1622 & $16 / 06 / 2000$ & $03: 28: 30$ & 3600 & 0.55 \\
1622 & $21 / 06 / 2000$ & $02: 50: 40$ & 3600 & $?$ \\
1622 & $21 / 06 / 2000$ & $03: 55: 12$ & 3600 & $?$ \\
1905 & $19 / 06 / 2000$ & $00: 43: 52$ & 3600 & 1.0 \\
1905 & $19 / 06 / 2000$ & $01: 45: 19$ & 3600 & 1.0 \\
1905 & $22 / 06 / 2000$ & $00: 32: 10$ & 3600 & 0.8 \\
1905 & $22 / 06 / 2000$ & $01: 33: 28$ & 3600 & 0.8 \\
201432 & $18 / 06 / 2000$ & $05: 00: 42$ & 3600 & 0.8 \\
201432 & $18 / 06 / 2000$ & $06: 05: 20$ & 3600 & 0.8 \\
201432 & $22 / 06 / 2000$ & $02: 38: 09$ & 3600 & 0.35 \\
201432 & $22 / 06 / 2000$ & $03: 41: 27$ & 3600 & 0.35 \\
202765 & $19 / 06 / 2000$ & $02: 50: 18$ & 3600 & 1.0 \\
202765 & $19 / 06 / 2000$ & $03: 51: 39$ & 5400 & 1.5 \\
202765 & $21 / 06 / 2000$ & $00: 42: 01$ & 3600 & 1.1 \\
202765 & $21 / 06 / 2000$ & $01: 43: 46$ & 3600 & $?$ \\
\hline
\end{tabular}

For all the observations the slit was $1^{\prime \prime}$ and the CCD binning $2 \times 2$.

arc lines in the region of the Li doublet was $0.015 \mathrm{~nm}$, providing a resolution $R \sim 45000$.

In spite of our efforts we managed to obtain only a moderate increase of the $S / N$ ratio, with respect to the pipeline data, showing that the quality of the pipeline data is very high and perfectly adequate for scientific analysis. In order to rectify the spectrum, removing the signature of the instrument response function, we used the flux standard Feige 67, which has been observed on June the 15th with the same spectrograph setting as the program stars, except for the slit width which was of $10^{\prime \prime}$. The flux data used to calibrate it, was that of Oke (1990). The wavelength scale of the Oke data is uncertain by about $0.6 \mathrm{~nm}$, we therefore arbitrarily shifted his data so that the position of $\mathrm{H} \alpha$ coincided with that in our spectrum. The quality of this relative flux-calibration is of the order of a few percent. This was estimated by comparing the flux of Feige 66, observed on June 20th and calibrated with the response function derived from the observations of Feige 67, to the flux of Oke (1990). Another spectrum of Feige 66, observed on June 17th is almost identical to that of June 20th, after a suitable scaling. This gives us confidence that the instrumental response function is very stable and reproducible. We stress that the purpose of this calibration is only to rectify the spectrum and it is meant to be accurate in a relative, not absolute sense. The flux calibration was not entirely successful in removing the ondulation in the

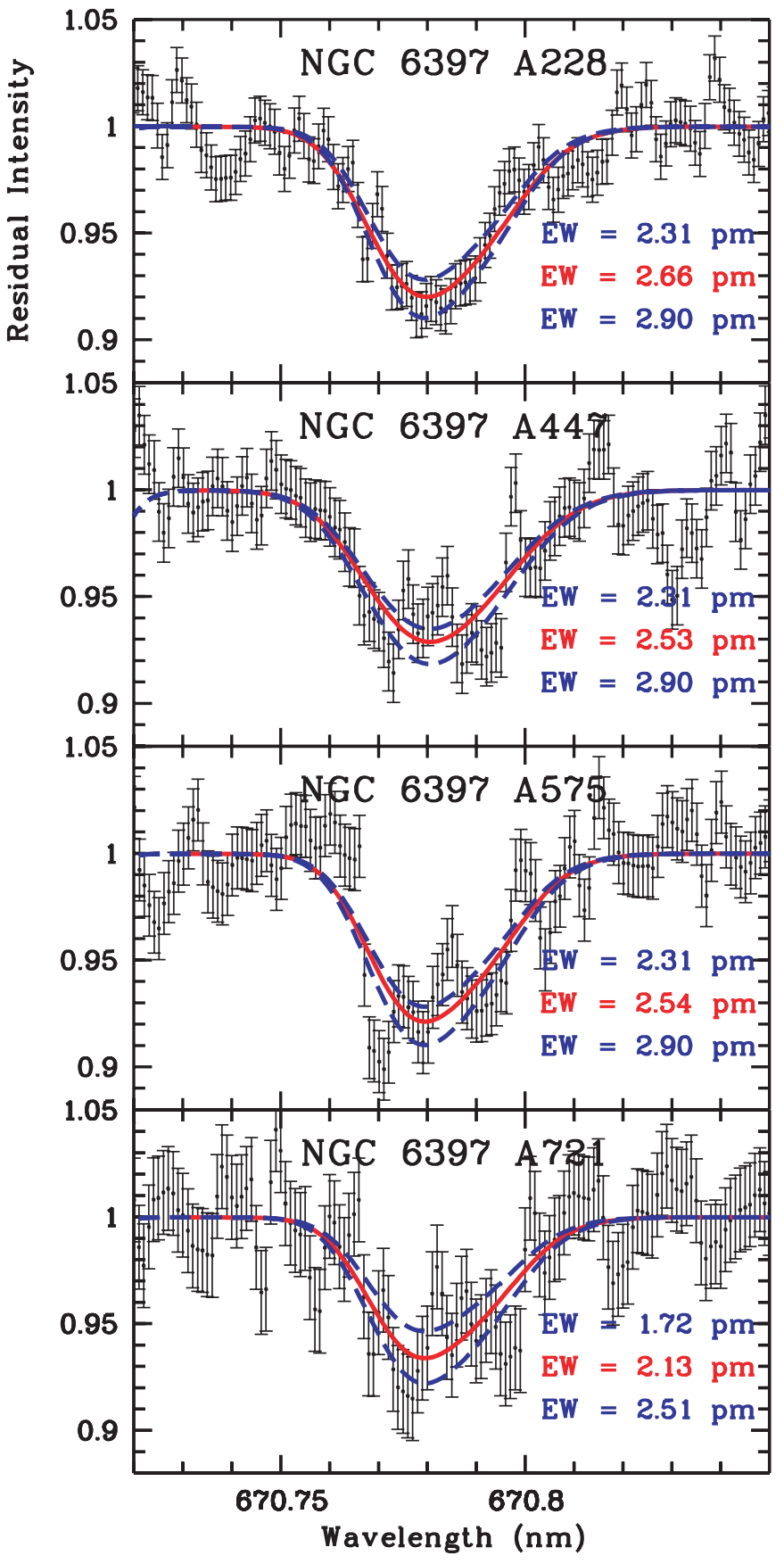

Fig. 2. Li doublet for four of the stars of Thévenin et al. (2001).

continuum; in the end we therefore applied a Fourier high-pass filter to remove it.

For each star we had four independent exposures which were independently calibrated, rectified and then coadded. The coadded spectrum was normalized around the Li doublet by fitting a spline through several continuum windows about $0.2 \mathrm{~nm}$ wide.

For the stars of Thévenin et al. (2001) the raw data and calibration frames were retrieved from the ESO-VLT archive and were reduced with exactly the same prescriptions as our own data. With respect to our own data we highlight the following differences of the Thévenin et al. (2001) data: 1) the 


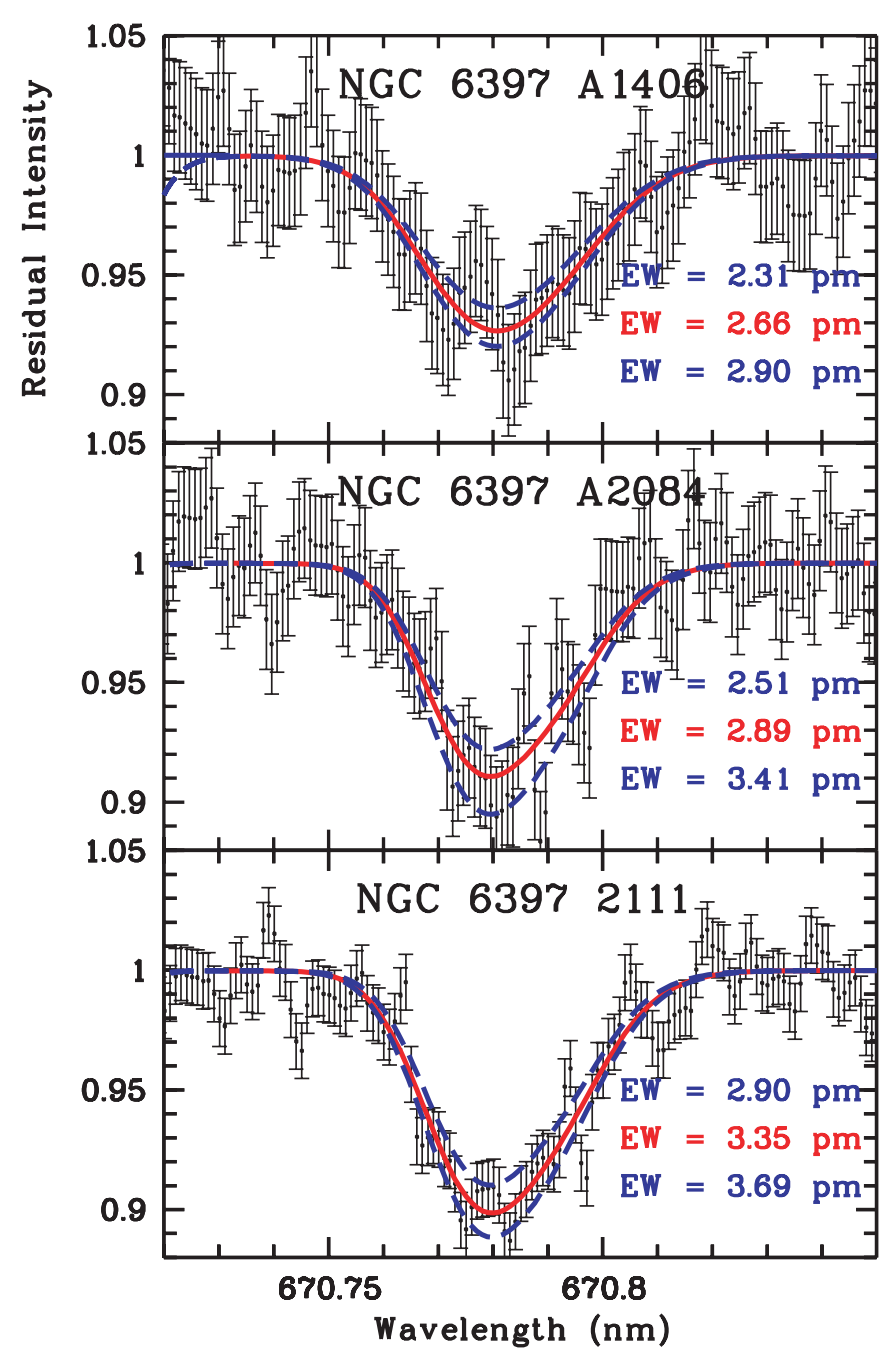

Fig. 3. Li doublet for three of the stars of Thévenin et al. (2001).

CCD binning which was $1 \times 1$; 2) the slit width was 1 .' 1 ; 3) the central wavelength was $580 \mathrm{~nm}$, which implies that the Li doublet falls on the MIT CCD; 4) there was no dichroic and observations were taken in the red arm only. The Thévenin et al. (2001) data has a slightly lower resolution and $S / N$, although a better sampling of the resolution element. The flux calibration was performed using the standard star LTT9239, observed on 05/06/2000 with the same instrument setup as the stars of NGC 6397. Like for our own data multiple spectra of the same star were coadded and normalized with a spline.

\section{Equivalent widths measurements}

In order to measure accurately the equivalent width of the $\mathrm{Li}$ doublet we used a least-squares minimization to determine the best fitting synthetic profile. Since the data has been rebinned to a constant wavelength step the theorems concerning the statistical properties of the $\chi^{2}$ do not apply. Nevertheless $\chi^{2}$ fitting may be used and the error conveniently estimated using Monte Carlo simulations (Bonifacio, in preparation).

To perform the minimization we used MINUIT (James 1998) using as fitting parameters equivalent width, broadening (assuming a Gaussian shape, which is appropriate for the

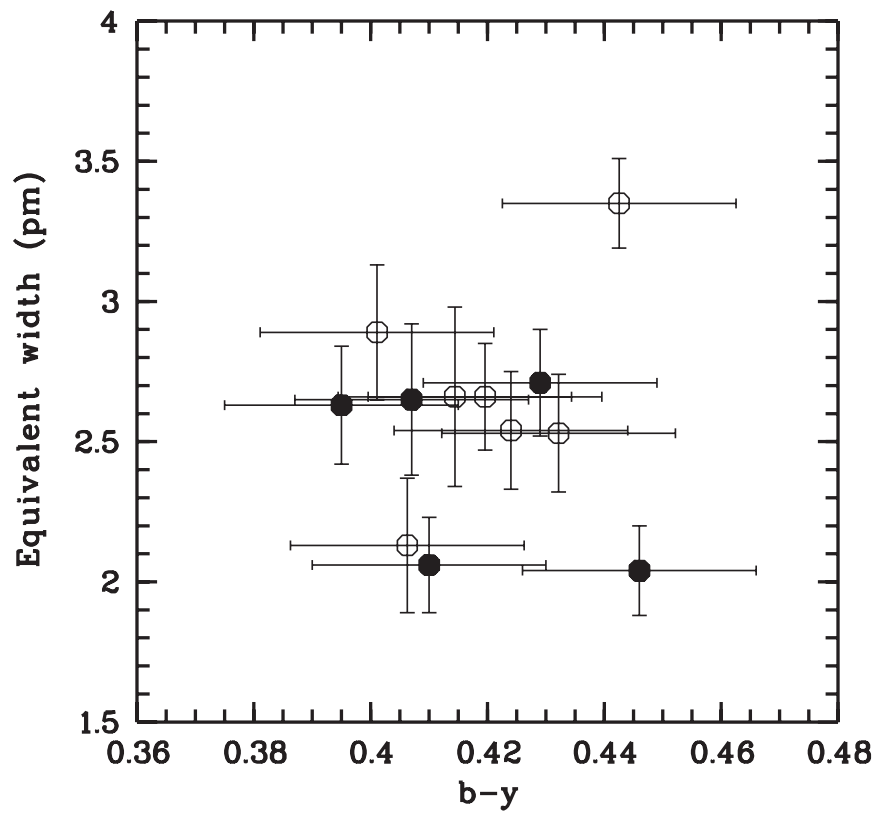

Fig. 4. $E W$ versus $b-y$. Filled symbols: stars observed by us; open symbols: stars observed by Thévenin et al. (2001).

UVES instrumental profile), wavelength shift and a multiplicative constant for continuum adjustment. To better constrain the continuum we included also two continuum stretches of $0.2 \mathrm{~nm}$ each to the blue and to the red of the line. The $S / N$ ratio was estimated from the root mean square deviation in these continuum windows and is given in Col. (6) of Table 2. The fitted profiles are shown in Figs. 1-3 together with two bracketing synthetic profiles. The equivalent widths of the fitted profiles in units of pm $\left(10^{-12} \mathrm{~m}\right)$ are given in Col. (2) of Table 2. It is customary to use the Cayrel formula (Cayrel 1988) to estimate the errors in equivalent widths. This formula assumes a gaussian line profile and depends on the full width at half-maximun of the line, on the pixel size (in wavelength units) and on the $S / N$ ratio. Since the Li doublet profile is non-gaussian we performed a Monte Carlo simulation to estimate the errors on equivalent widths. For each star we took its best fitting synthetic profile, added Poisson noise to obtain the observed $S / N$ ratio and performed the fitting several hundreds times. The standard deviation of the fitted equivalent widths was then taken as the $1 \sigma$ error on the measured equivalent width. The derived values are given in Col. (3) of Table 2, the estimates from the Cayrel formula are given in Col. (5). This shows that the Cayrel formula does provide a reasonable estimate of the error. We adopt the results of the Monte-Carlo simulations as error estimates. In Col. (4) of Table 2 we also provide the equivalent widths obtained by fitting a Gaussian to the data (we used the iraf task splot). One may easily see that the two measures are consistent within errors. In fact at this resolution and $S / N$ approximating the doublet with a single Gaussian is acceptable. 
Table 2. Equivalent widths and Li abundances for TO stars in NGC 6397.

\begin{tabular}{|c|c|c|c|c|c|c|c|c|c|c|c|}
\hline star \# & $\begin{array}{l}E W \\
\mathrm{pm}\end{array}$ & $\begin{array}{c}\sigma_{\mathrm{MC}} \\
\mathrm{pm}\end{array}$ & $\begin{array}{c}E W_{\mathrm{g}} \\
\mathrm{pm}\end{array}$ & $\begin{array}{l}\sigma_{\mathrm{C}} \\
\mathrm{pm}\end{array}$ & $S / N$ & $\begin{array}{r}A(\mathrm{Li}) \\
T=6476 \mathrm{~K}\end{array}$ & $\sigma_{\mathrm{Li}}$ & $\begin{aligned} & T_{\mathrm{V}} \\
T= & T_{\mathrm{V}}\end{aligned}$ & $\Delta T_{\mathrm{V}}$ & $A(\mathrm{Li})$ & $\sigma_{\mathrm{Li}}$ \\
\hline (1) & (2) & (3) & (4) & (5) & (6) & (7) & (8) & (9) & (10) & (11) & (12) \\
\hline \multicolumn{12}{|c|}{ measures from our VLT-UVES data } \\
\hline 1543 & 2.63 & 0.21 & 2.60 & 0.21 & 85 & 2.41 & 0.07 & 6401 & 30 & 2.36 & 0.05 \\
\hline 1622 & 2.04 & 0.16 & 2.01 & 0.18 & 105 & 2.29 & 0.07 & 6394 & 32 & 2.24 & 0.05 \\
\hline 1905 & 2.71 & 0.19 & 2.62 & 0.21 & 85 & 2.43 & 0.07 & 6456 & 9 & 2.41 & 0.04 \\
\hline 201432 & 2.06 & 0.17 & 2.11 & 0.21 & 131 & 2.29 & 0.07 & 6427 & 23 & 2.26 & 0.05 \\
\hline 202765 & 2.65 & 0.27 & 2.77 & 0.30 & 92 & 2.42 & 0.08 & 6394 & 32 & 2.36 & 0.06 \\
\hline \multicolumn{12}{|c|}{ measures from archive VLT-UVES data } \\
\hline A228 & 2.66 & 0.19 & 2.61 & 0.19 & 95 & 2.42 & 0.07 & 6274 & 58 & 2.28 & 0.06 \\
\hline A447 & 2.53 & 0.21 & 2.66 & 0.25 & 73 & 2.39 & 0.07 & 6374 & 37 & 2.33 & 0.05 \\
\hline A575 & 2.54 & 0.21 & 2.52 & 0.27 & 69 & 2.40 & 0.07 & 6362 & 40 & 2.32 & 0.05 \\
\hline A721 & 2.13 & 0.24 & 2.30 & 0.36 & 51 & 2.31 & 0.08 & 6386 & 34 & 2.25 & 0.06 \\
\hline A1406 & 2.66 & 0.34 & 2.84 & 0.42 & 43 & 2.42 & 0.09 & 6345 & 44 & 2.37 & 0.07 \\
\hline A2084 & 2.89 & 0.23 & 2.88 & 0.38 & 48 & 2.46 & 0.07 & 6383 & 35 & 2.38 & 0.05 \\
\hline A2111 & 3.35 & 0.16 & 3.29 & 0.21 & 86 & 2.53 & 0.06 & 6207 & 69 & 2.33 & 0.06 \\
\hline \multicolumn{12}{|c|}{ measures of Pasquini \& Molaro (1996) from NTT-EMMI data } \\
\hline A611 & 2.60 & 0.80 & & & & & & 6371 & 29 & 2.34 & 0.18 \\
\hline C4602 & 3.40 & 0.67 & & & & & & 6306 & 39 & 2.42 & 0.11 \\
\hline A853 & 4.30 & 0.50 & & & & & & 6206 & 70 & 2.48 & 0.08 \\
\hline
\end{tabular}

\section{Li abundances}

To determine the Li abundances we fitted the equivalent widths measured as described in the previous section to those computed with the SYNTHE code (Kurucz 1993), as described in Bonifacio \& Molaro (1997). The atmospheric parameters are the same adopted in Gratton et al. (2001), namely $T_{\text {eff }}=$ $6476 \mathrm{~K} ; \log g=4.10$ and $\xi=1.32 \mathrm{~km} \mathrm{~s}^{-1}$ and a metallicity of -2.0 . An LTE model atmosphere with these parameters was computed with the ATLAS 9 code (Kurucz 1993) using $\alpha$ enhanced opacity ${ }^{1}$ distribution functions with a microturbulent velocity of $1 \mathrm{~km} \mathrm{~s}^{-1}$ and with the overshooting option switched off. The results are given in Col. (7) of Table 2. In Col. (8) of the Table we provide also the errors in $\mathrm{Li}$ abundance obtained by summing quadratically the errors arising from equivalent widths and from effective temperatures; as error in $T_{\text {eff }}$ we assumed $90 \mathrm{~K}$, as given in Gratton et al. (2001). For this effective temperature the correction for Li depletion predicted by the standard isochrones of Deliyannis et al. (1990) is zero. The correction for NLTE effects computed by Carlsson et al. (1994) is +0.01 dex.

Although the assumption that all the stars share the same temperature is acceptable to determine the metallicity and the general abundance pattern of the stars, it may prove to be somewhat crude to discuss the dispersion of $\mathrm{Li}$ abundances. In

\footnotetext{
1 We note that at this low metallicity the differences between models with and without $\alpha$-enhancement are negligible as shown e.g., by Gratton et al. (2000).
}

Gratton et al. (2001) we derived the effective temperature by fitting the mean $\mathrm{H} \alpha$ profile, obtained by averaging all the spectra of the different stars, on the assumption that they share the same temperature. To derive temperatures for each star one could fit the individual $\mathrm{H} \alpha$ profiles, however, the relatively poor $S / N$ of the spectra would not allow to derive temperatures with an accuracy better than $150 \mathrm{~K}$. Another possibility is to use $(b-y)_{0}$, but this is even worse: a difference of $(b-y)_{0}$ of 0.015 mag translates into a temperature difference of about $130 \mathrm{~K}$. The photometric error alone is of this order of magnitude. Figure 4 shows quite clearly that there is no correlation between $(b-y)$ and Li equivalent width, which should be the case if $(b-y)$ is tightly correlated with temperature and the stars have more or less the same Li abundance. Instead our extensive photometry allows us to derive quite accurately the mean locus of the cluster isochrone. What is shown in Fig. 5 is an average with outliers eliminated using an iterative clipping procedure.

It is clear that all the stars observed are slightly brighter than the turn-off where a tight one to one relation holds between $V$ an $b-y$. Adopting the average reddening of $E(b-y)=0.137$, which we derived comparing colours and temperatures of cluster and field stars (Gratton et al. 2001), we may thus map $V$ onto $(b-y)_{0}$ and hence temperature through comparison with the theoretical colors given by Kurucz (1993). These temperatures were corrected to be on the same scale as those from $\mathrm{H} \alpha$, exactly as done for the field stars in Gratton et al. (2001). We recall that this temperature scale agrees quite well with the IRFM temperature scale (Alonso et al. 1996). We have applied 
Table 3. Equivalent widths for field stars.

\begin{tabular}{|c|c|c|c|c|c|c|c|c|c|c|c|}
\hline star & $\begin{array}{l}E W \\
\text { pm } \\
(2)\end{array}$ & $\begin{array}{r}\sigma_{E W} \\
\mathrm{pm} \\
(3)\end{array}$ & $\begin{array}{r}{[\mathrm{Fe} / \mathrm{H}]} \\
\operatorname{dex} \\
(4)\end{array}$ & $\begin{array}{r}\log g \\
\operatorname{cgs} \\
(5)\end{array}$ & $\begin{array}{r}T_{\mathrm{H} \alpha} \\
\mathrm{K} \\
(6)\end{array}$ & $\begin{array}{r}T_{\mathrm{F} 94} \\
\mathrm{~K} \\
(7)\end{array}$ & $\begin{array}{r}T_{\text {IRFM }} \\
\mathrm{K} \\
(8)\end{array}$ & $A(\mathrm{Li})$ & $\begin{array}{l}\sigma_{\mathrm{Li}} \\
(10)\end{array}$ & $\begin{array}{r}A(\mathrm{Li}) \\
\mathrm{Tc} \\
(11)\end{array}$ & $\begin{array}{r}\mathrm{A}(\mathrm{Li}) \\
\mathrm{TcNLTE} \\
(12)\end{array}$ \\
\hline HD 108177 & 3.37 & 0.09 & -1.70 & 4.44 & 6027 & 6090 & 6067 & 2.25 & 0.11 & 2.28 & 2.29 \\
\hline HD 116064 & 3.10 & 0.09 & -1.87 & 4.35 & 6192 & 5822 & 5923 & 2.30 & 0.12 & 2.31 & 2.32 \\
\hline HD 140283 & 4.95 & 0.06 & -2.46 & 3.67 & 5898 & 5814 & 5691 & 2.31 & 0.12 & 2.36 & 2.38 \\
\hline HD 166913 & 3.66 & 0.13 & -1.59 & 4.08 & 5921 & 5955 & 6020 & 2.21 & 0.11 & 2.25 & 2.26 \\
\hline HD 181743 & 3.78 & 0.09 & -1.81 & 4.42 & 6130 & & 5927 & 2.36 & 0.12 & 2.38 & 2.39 \\
\hline
\end{tabular}

this calibration to four field stars with neglegible reddening, colours similar to our TO stars and metallicities in the range $-2.2 \leq[\mathrm{Fe} / \mathrm{H}]-1.8$, and found a mean difference of $7 \mathrm{~K}$ between these temperatures and IRFM temperatures. With this calibration an error of $0.05 \mathrm{mag}$ in $V$ translates into an error in the range $20-70 \mathrm{~K}$. These temperatures are given in Col. (9) of Table 2 and the errors deriving from an error 0.05 mag are given in Col. (10). This estimate is somewhat conservative for the photometric error alone, however if there is any variation of reddening, even at the level of $0.01 \mathrm{mag}$ in $E(B-V)$, this translates into differences of $0.03 \mathrm{mag}$ in $V$. These temperature errors should be interpreted as "internal errors", thus appropriate to assess the issue of dispersion in $\mathrm{Li}$ abundances among the cluster stars. The "external error" which takes into account the systematic error of our adopted temperature calibration is difficult to assess, since it may depend on many different sources, given that the calibration involves several different steps, each with its systematic error. However since we have shown we are on the same scale of Alonso et al. (1996) we may assume the error of this scale as systematic error. This amounts to $80 \mathrm{~K}$ in this temperature range.

The Li abundances derived assuming these temperatures are given in Col. (11) of Table 2, and their associate errors in Col. (12). For these temperatures the correction for Li depletion predicted by the isochrones of Deliyannis et al. (1990) is zero for all but the two coolest stars, for which it is of the order of 0.01 dex. The NLTE corrections are also small and all of the order of +0.01 dex for all stars.

\section{Discussion}

\subsection{Observed dispersion of Li abundances}

If we adopt the same temperature for all the stars of NGC 6397 the mean $\mathrm{Li}$ abundance is $A(\mathrm{Li})=2.39^{2}$, with a standard deviation of $0.07 \mathrm{dex}$, or $A(\mathrm{Li})=2.40$, once NLTE effects are accounted for according to Carlsson et al. (1994). The question is whether such a scatter is consistent with the observational errors or if it is larger. The mean error is $0.074 \mathrm{dex}$, which intuitively suggests that there is no extra scatter. A KolmogorovSmirnov test of the null hypothesis that the data do not come from a normal distribution with mean 2.39 and standard deviation 0.074 gives a probability of $\sim 0.32$, thus rejecting the

\footnotetext{
${ }^{2}$ on the usual scale $A(\mathrm{Li})=\log [N(\mathrm{Li}) / N(\mathrm{H})]+12$.
}

hypothesis. One could argue that the Kolmogorov-Smirnov cannot be applied here since the measures have different errors and therefore cannot have been drawn from the same parent distribution. We therefore test the hypothesis that the observed dispersion is simply due to the observational errors by a straightforward Monte Carlo simulation. We generate 1000 sets of 12 Gaussian random numbers with mean 2.39 and standard deviation equal to the error estimate of each data point. For each realization of 12 "observations" we compute the standard deviation $s$. The mean value of $s$ over the sample is 0.073 with a standard deviation of 0.015 . Hence the data is consistent with the notion that the stars share the same Li abundance, the dispersion being entirely due to observational error. The error on $\mathrm{Li}$ abundances is, in fact, largely dominated by the error in $T_{\text {eff }}$. A spread in effective temperature of $120 \mathrm{~K}$, neglecting any error in the measurement of equivalent widths, would provide a spread in Li abundances of 0.08 dex, slightly larger than the observed spread. This suggests that the error on the effective temperature is in fact 10 or $20 \mathrm{~K}$ larger than estimated in Gratton et al. (2001), which is not unreasonable. It is therefore likely that the stars do not share precisely the same effective temperature, as assumed in Gratton et al. (2001).

Let us therefore consider the sample with $\mathrm{Li}$ abundances derived assuming the temperatures from the $V-T_{\text {eff }}$ calibration. It is apparent from the plot in Fig. 6 that there is very little scatter. In fact also the three stars observed with NTT by Pasquini \& Molaro (1996) are totally consistent with the present data set, once we adopt the same temperature scale. The mean $\mathrm{Li}$ abundance is 2.32 with a standard deviation of 0.056 dex. To verify whether this is compatible with the estimated errors we resort again to a Monte Carlo simulation. The mean standard deviation of 1000 samples is 0.051 with a standard deviation of 0.011 . Thus also with this temperature scale, in spite of the very small errors adopted for the temperatures there is no evidence of any intrinsic scatter. In fact we may use these results to place an upper limit on the maximum intrinsic scatter allowed. In order to be not detected at $1 \sigma$ the intrinsic scatter should have been such that, summed quadratically to the mean scatter provided by observational errors (estimated as 0.051 from the Monte Carlo simulation) it does not exceed this mean by more than $1 \sigma$. Let $\delta(\mathrm{Li})$ be the intrinsic scatter produced by the Li depletion mechanism, one has $\delta(\mathrm{Li})<\sqrt{0.062^{2}-0.051^{2}} \approx 0.035$. 


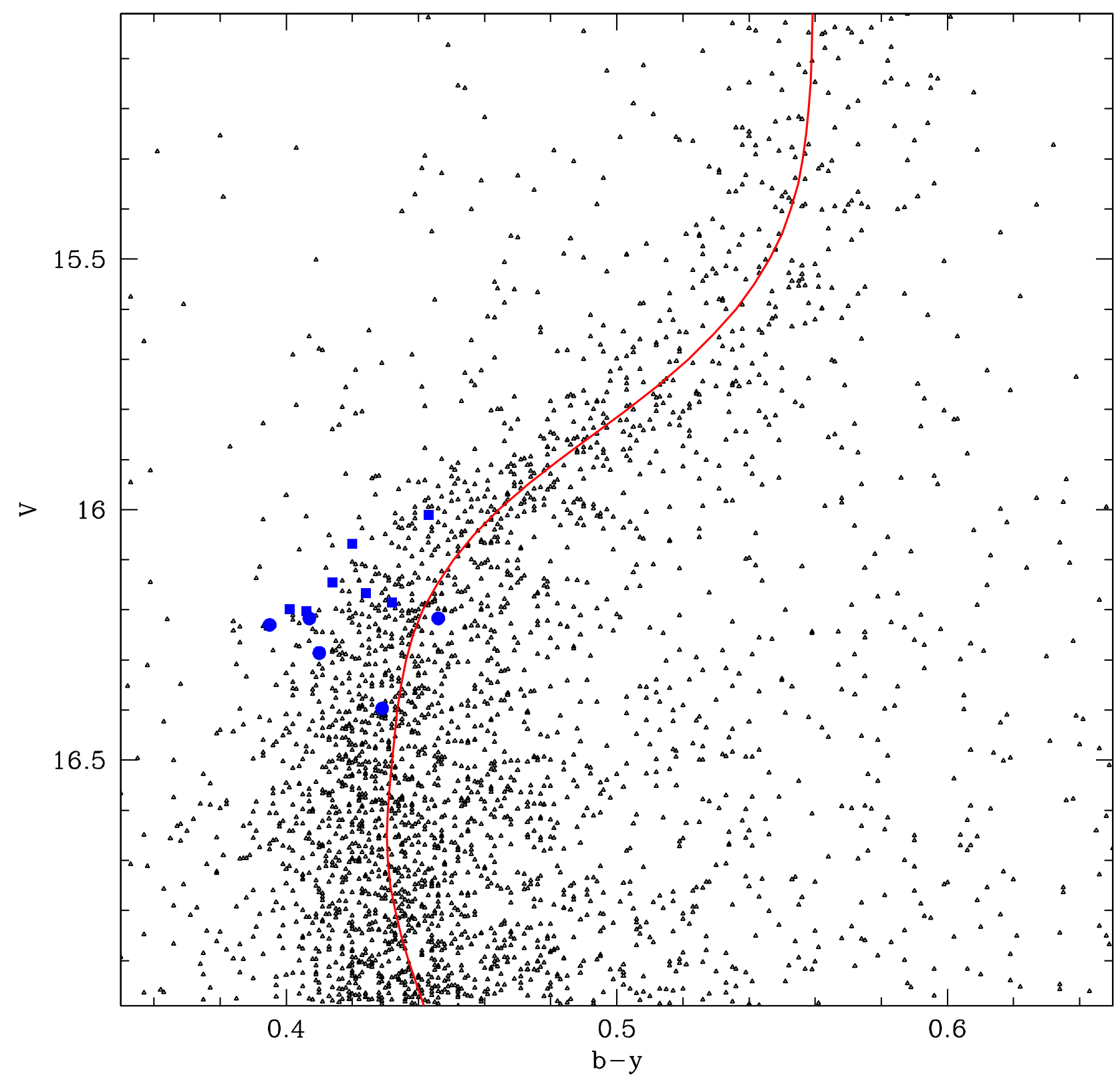

Fig. 5. Color-magnitude diagram of NGC 6397, where our own observations have been calibrated using 3500 stars in common with AnthonyTwarog \& Twarog (2000). Filled circles are the stars for which we obtained UVES spectra and filled squares are those observed by Thévenin et al. (2001).

\subsection{Comparison with previous results}

Pinsonneault et al. (1999) reported unpublished results by Thorburn and collaborators which showed a significant dispersion of $0.3-0.5$ dex in $\mathrm{Li}$ abundances in this cluster, based on spectra of about 20 stars, but since these results have not been published in any detailed form we cannot make any hypothesis on the reason for the discrepancy with the present results.

The difference between the Li abundances given here and those of Thévenin et al. (2001) lies only in the different effective temperatures adopted. Although Thévenin et al. (2001) do not give the $\mathrm{Li}$ equivalent widths we used their $\mathrm{Li}$ abundances and their effective temperatures to compute the equivalent widths of the Li doublet for their stars, and these were always within $0.2 \mathrm{pm}$ of our measured equivalent widths, as expected. If we compare the colours for the two sets of stars, by estimating for the Thévenin et al. stars $(b-y) \approx 0.74(B-V)$, we find that, although the Thévenin et al. stars are about 0.10.2 mag brighter, thus slightly more evolved and, on average, cooler, they span the same colour range as our stars. In Fig. 4 we show equivalent widths for our stars (filled symbols) and for those of the Thévenin et al. (2001) stars (open symbols) as a function of $(b-y)$. It is clear that the two sets of stars occupy the same area in this plane.

From Fig. 6 it is clear that our measures are in good agreement with those of Pasquini \& Molaro (1996) and the difference in $\mathrm{Li}$ abundances is due to the different temperature scales adopted. 


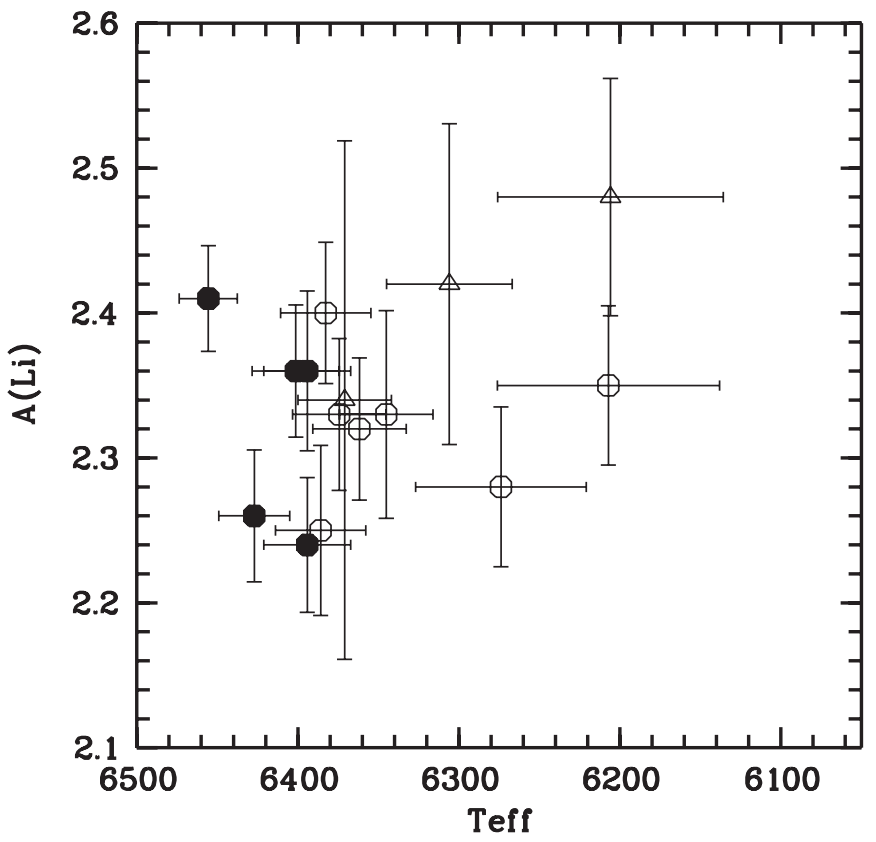

Fig. 6. Li abundances as a function of effective temperatures derived from the location of stars along the cluster mean locus. The four stars observed by us are shown as filled circles, those observed by Thévenin et al. (2001) as open symbols, while the three stars observed by Pasquini \& Molaro (1996) are shown as open triangles.

\subsection{Comparison with field stars}

Several halo field stars have been observed at twilight in the course of the program and their atmospheric parameters, determined as done for cluster stars are reported in Gratton et al. (2001). The effective temperatures are different from those of Gratton et al. (2001) due to the better straightening of the spectra achieved here thanks to the flux calibration. To compare the Li content of NGC 6397 with that of field stars we select only those with metallicity below $[\mathrm{Fe} / \mathrm{H}]=-1.5$, which is roughly the value where $\mathrm{Li}$ abundances begin to rise off the Spite plateau, and effective temperature higher than 5700, which is roughly the value below which Li depletion due to convection becomes non-negligible. In Table 3 we give the equivalent widths of the Li doublet as measured from our spectra, the effective temperatures derived from the $\mathrm{H} \alpha$ profiles and for reference, the effective temperatures of Fuhrmann et al. (1994), also based on Balmer lines, and of Alonso et al. (1996), based on the Infrared Flux Method (IRFM). The observed field stars are somewhat cooler than our cluster stars. As noted by Bonifacio \& Molaro (1997) at temperatures below $6300 \mathrm{~K}$, even the standard models of stellar evolution predict a slight Li depletion (Deliyannis et al. 1990). Thus to make a meaningful comparison we corrected the $\mathrm{Li}$ abundances for the depletion predicted by the isochrones of Deliyannis et al. (1990) and these corrected values are given in Col. (11) of Table 3: the corrections are small for all the stars. Also the correction for NLTE effects, albeit small, is temperature dependent (Carlsson et al. 1994) we thus corrected also for this effect and the corrected values are given in Col. (12) of Table 3. As described above we applied the same corrections also to the stars of NGC 6397.
The star HD 116064 has been noted to be a double-lined spectroscopic binary by Smith et al. (1998) and our spectrum does indeed confirm the duplicity. The veiling is unknown, however the star's Li abundance is comparable with that of the other stars, suggesting that at these wavelengths it is neglegible. The mean $A(\mathrm{Li})$ for the five field stars is 2.29 with a standard deviation of 0.058 , in excellent agreement with the mean $A(\mathrm{Li})=2.32$ for the $12 \mathrm{TO}$ stars of NGC 6397. If we consider the depletion corrected values, the mean $\mathrm{Li}$ abundance is 2.31 for the field stars and 2.32 for the NGC 6397 sample. When considering also the NLTE corrections it becomes 2.33 for the field stars and 2.34 for the cluster stars. We believe there is no need for any sophisticated statistical analysis to conclude that the Li abundance in NGC 6397 is the same as that of the field stars, within the stated errors, supporting the primordial origin of $\mathrm{Li}$ in these stars.

Preliminary results of the analysis of a high quality sample of field stars with IRFM temperatures have been reported by Bonifacio (2001) and the current best estimate of the plateau $\mathrm{Li}$ abundance is $2.317 \pm(0.014)_{1 \sigma} \pm(0.05)_{\text {sys. }}$. This value happens to be in perfect agreement with the values found here both for field stars and for NGC 6397 TO stars.

\subsection{Evolutionary models and Li depletion}

There is a long-standing debate concerning Li depletion on the Spite plateau. If $\mathrm{Li}$ depletion is possible, the pristine $\mathrm{Li}$ abundance is higher than the observed. As noted above, standard models of stellar evolution cannot account for any significant depletion in dwarf stars. Early attempts to compute more sophisticated models including diffusion (Michaud et al. 1984) or rotation-induced turbulence (Vauclair 1988) predicted large depletions ${ }^{3}$ of up to a factor of 10 . These models predicted a considerable scatter in the observed $\mathrm{Li}$ abundances and also other features, such as a slope of the plateau with effective temperature and metallicity, or an enhanced $\mathrm{Li}$ abundance among post-TO stars. All of these effects have been ruled out by the observations.

The situation on the theoretical side has dramatically changed in the last year: new sets of models seem to be able to produce a plateau with very little scatter. The rotationally mixed models of Pinsonneault et al. (2002), the diffusive models of Salaris \& Weiss (2001) and the models of Théado \& Vauclair (2001), which include rotational-mixing, diffusion and compositions gradients, are all capable of reproducing a plateau, with a very small intrinsic dispersion. It must be however noted, that large depletion factors are not any longer found, and the above papers predict primordial $\mathrm{Li}$ abundances in good agreement with the best current estimates of the baryonic density of the universe ${ }^{4}$. The intrinsic dispersions allowed for by the various depletion mechanisms, are somewhat larger than

\footnotetext{
${ }^{3}$ For the purpose of the present discussion we consider Li to be depleted, even if it is not destroyed, but simply removed from the observable layers of the stars. What is relevant is that the true $\mathrm{Li}$ abundance is higher than that deduced from the analysis of the spectrum.

${ }^{4}$ Pinsonneault et al. (2002) derive $A(\mathrm{Li})=2.4$, Salaris \& Weiss (2001) and Théado \& Vauclair (2001) derive $A(\mathrm{Li})=2.5$.
} 
the maximum value of 0.035 dex derived above; it is unlikely, however, that this value can rule out any of them. A common feature of all models which predict Li depletion is the presence of a small number of "outliers", i.e. stars with a Li abundance more than $3 \sigma$ below the mean and such objects are indeed known to exist among field stars. It may therefore be of some significance that out of 15 TO stars observed in NGC 6397 none appears to be significantly Li depleted. We mantain that there is no compelling evidence in favour of any of the proposed depletion mechanisms, although none can be ruled out. Economy of hypothesis suggests that standard models with no Li depletion should be preferred. In spite of this there are strong arguments in favour of the presence of both diffusion and rotational mixing, although, admittedly, they come mostly from the study of the Pop I.

Helioseismic studies of the Sun imply that atomic diffusion is important (Guenther et al. 1996). Theoretical investigations show that it should be important also in metal-poor stars (Michaud et al. 1984; Castellani et al. 1997; Salaris \& Weiss 2001). Lebreton et al. (1999) have shown that the H-R diagram of field stars with accurate parallaxes, in the metallicity range $-1.0 \leq+0.3$, is best fitted when models which include diffusion are used. All diffusive models predict that surface lithium sinks below the bottom of the thin surface main sequence convection zone, thus becoming unobservable, i.e. depleted, in our parlance. Two features are predicted by diffusive models: excess dispersion and a downturn at the hot end of the plateau; both are in disagreement with the observations. To alleviate the problem it has been suggested that a modest mass-loss (Vauclair \& Charbonnel 1995; Swenson 1995) may undo the sedimentation predicted by diffusion. These mass loss models, however, also predict a down-turn of Li abundances at the hot edge of the Spite plateau (Vauclair \& Charbonnel 1995) and a constant $\mathrm{Li}$ abundance among cooler sub-giants (Swenson 1995), but none of these features is observed. Furthermore there is no observational evidence that such a mass-loss actually occurs. Recently Vauclair (1999) has proposed a model in which meridional circulation, in presence of a composition gradient, created by diffusion, could lead to a quasi-equilibrium stage in which diffusion and circulation are both inhibited. The detailed results for these model and the impact on the Spite plateau have been discussed by Théado \& Vauclair (2001). The predicted dispersion in the plateau is very small, however there persists a slight downturn at high temperatures and an increased dispersion. Similar results are found by Salaris \& Weiss (2001), using purely diffusive models (no rotational mixing and no composition gradients): a very uniform plateau, with a slight downturn, and excess dispersion at the hottest edge, above $6200 \mathrm{~K}$ (see their Figs. 7 and 8). This is exactly the region covered by our observations and no downturn or extra dispersion are observed. Admittedly our sample, of 12 stars only, is very small, and Salaris \& Weiss (2001) predict, based on their Monte Carlo simulations, that at least 40-50 stars at the hot end of the Spite plateau should be observed in order to detect the downturn. Such a number of stars shall be easily available in the near future, with the FLAMES instrument, but for the time being we conclude that our results for NGC 6397 do not support neither the models of Théado \& Vauclair (2001) nor those of Salaris \& Weiss (2001). The Li abundance in NGC 6397 is constant and is the same as that of field stars of much lower $T_{\text {eff. }}$. Also the iron abundance of the TO stars (Gratton et al. 2001; Thévenin et al. 2001) is equal to that of sub-giant and giant stars (Gratton et al. 2001; Castilho et al. 2000). Instead diffusive models predict the photospheric metallicity of a star to constantly decrease, due to diffusion, during its main sequence lifetime, reaching a minimum around the turn-off and then increasing back again to the original metallicity along the red giant branch (Castellani et al. 1997). The observational evidence presented here and in Gratton et al. (2001) is against any significant sedimentation due to diffusion in the stars of NGC 6397 , both for $\mathrm{Li}$ and $\mathrm{Fe}$.

The strongest evidence of the inadequacy of the standard models probably comes from the study of lithium in open clusters; among them, the presence of the Li dip first discovered in the Hyades (Boesgaard \& Tripicco 1987), the observed scatter in $\mathrm{Li}$ abundance among the solar type stars in the field and M 67 (Pasquini et al. 1994; Pasquini et al. 1997), require that additional mixing mechanisms, besides convection, are at work. Rotational mixing models have the ingredients to reproduce several of the observed features, and, although they fail in the quantitative reproduction of the observations (see e.g. Randich et al. 2000; Randich et al. 2002), they are promising, expecially when considering stars with high masses and large rotational velocities such those around the Li dip. Open cluster data has been interpreted with models with different physical ingredients: Pasquini et al. (2001) used the Charbonnel \& Talon (1999) models, which include both rotational mixing and diffusion, for the interpretation of the Li observations of the intermediate age cluster NGC 3680; Boesgaard \& King (2002) used the Deliyannis \& Pinsonneault (1997) models, which include only rotational mixing, for the interpretation of the Hyades $\mathrm{Li}$ and Be data. Therefore there is no "non standard" set of models which is preferred for the explanation of Li observations in Pop I stars. We point out that the Pop II stars are of much lower metallicity and mass. We therefore do not see any compelling argument for the use of "non standard" models, in the case of Pop II stars. The only observable prediction we could verify is that they predict the presence of some "outlier" on a large sample; in a sample of 15 NGC 6397 turnoff stars we do not find any outlier, and this could be therefore taken as a mild indication against extra mixing predicted by "non standard" models. From an observational point of view we wish to add that Lucatello \& Gratton (2002) showed that the projected rotational velocities of our stars are less than $1.7 \mathrm{~km} \mathrm{~s}^{-1}$, which provides an additional constraint, to be incorporated in the comparison of theoretical and observational data.

\subsection{Normal Li and enhanced N: A paradox?}

The subgiant stars of the cluster show very strong $\mathrm{CN}$ bands. The full analysis of this data shall be presented elsewhere, however preliminary results suggest a conspicuous $\mathrm{N}$ enhancement of the order of 1 dex or more. This suggests that the material has been processed through the CNO cycle. Since our current understanding of subgiant stars precludes any mixing to occur 
in the star itself one is forced to conclude that the star was either formed from, or polluted by, such processed material. One should therefore expect that the TO stars share the same characteristics, although the $\mathrm{CN}$ bands are too weak to be detected and no direct information on $\mathrm{N}$ abundance is available. Here lies the paradox: if the material of these stars has been processed at the high temperatures where the CNO cycle is operating, Li should have been destroyed.

Such a paradox has been known to exist for many years for the N-rich metal-poor dwarfs, like HD 166913, studied also in this paper, which are Li - normal (Spite \& Spite 1986). In two of these stars Beveridge \& Sneden (1994) have found the neutron capture elements to be moderately enhanced and concluded that the most likely source of $\mathrm{N}$ and neutron capture elements was material provided by a thermally pulsing AGB star. In the stars of NGC 6397, instead, $[\mathrm{Sr} / \mathrm{Fe}] \sim-0.1$, $[\mathrm{Ba} / \mathrm{Fe}] \sim-0.2$ and $[\mathrm{Eu} / \mathrm{Fe}] \sim 0.4$, as shall be discussed elsewhere. Beveridge \& Sneden (1994) also argued that the N enhancement in halo dwarfs is unrelated to the phenomenon observed in N-, Na-, Al-rich, O-poor globular cluster giants, on the grounds that oxygen is not depleted in N-rich dwarfs. The abundance ratio of oxygen and magnesium to iron in this cluster is rather low $\sim+0.2$. Both oxygen and magnesium deficiencies could be explained by presence of material processed through complete $\mathrm{CNO}$ cycle. We add that $\mathrm{Al}$ enrichment is very moderate or absent in N-rich dwarfs, at the level of $[\mathrm{Al} / \mathrm{Fe}] \sim 0.2$ at most (Beveridge \& Sneden 1994; François 1986). Our TO stars in NGC 6397 show a similar low Al enhancement.

Our current understanding of $\mathrm{N}$ production require that it is synthetized in intermediate mass stars through CNO cyclying (Henry et al. 2000). However the simple picture by which Li is totally destroyed under these condition is not confirmed by recent computations. Ventura et al. (2001) studied the evolution of $\mathrm{N}$ and $\mathrm{Li}$ in metal-poor massive AGB stars, $\mathrm{CNO}$ processing at the bottom of the convective envelope overproduces nitrogen by $\sim 1.5 \mathrm{dex}$; lithium is initialy produced by the CameronFowler mechanism (Cameron \& Fowler 1971) and then destroyed, but a small amount of matter very Lithium rich is recycled to the interstellar medium. The net result is a reduction of $\sim 0.6$ dex for stars of metallicity comparable to that of NGC 6397. Note that a mass loss rate four times larger than the one assumed (which is within the uncertainty in the mass loss calibration) would leave the lithium essentially unaltered. Chieffi et al. (2001) have computed models for the evolution of zero metallicity stars. Such intermediate mass stars are capable of producing primary $\mathrm{N}$ and the most massive ones experience a normal AGB evolution, including thermal pulses and third dredge-up. What is interesting for us here, is that also in this case there is $\mathrm{Li}$ production via the Cameron-Fowler mechanism. We conclude that there are strong theoretical indications that at low metallicities $\mathrm{N}$ production is always accompanied by some Li production, so that $\mathrm{Li}$ is never totally depleted in the processed material. Mixing such material with material having the big bang lithium abundance would dilute nitrogen leaving it overabundant, but would not touch significantly lithium. Whether this is indeed a viable explanation depends very much on the precise degree of nitrogen enhance- ment. This may somehow alleviate the paradox, however some fine tuning is still needed in order to produce a Li abundance exactly at the level of the Spite plateau.

We are not here in a position to solve this puzzle, however we conclude this section by reasserting what we believe to be a sound observational fact: whichever the cause of these abundance anomalies, it cannot have altered significantly the Li content of the cluster stars.

\section{Conclusions}

The equivalent widths of the Li doublet of the 12 turn-off stars of NGC 6397 observed with VLT+UVES are consistent with the hypothesis that the stars all share the same Li abundance, the spread being entirely explained by observational error. The stars do not have exactly the same temperature but span a range of almost $300 \mathrm{~K}$. We thus consider the chemical homogeneity of this cluster, which we found for iron abundances in Gratton et al. (2001), to be firmly established. Why a globular cluster like NGC 6397 should show a constant Li abundance, with essentially no intrinsic scatter, while M 92, M 71 and M 13 show a scatter is puzzling. We note here however, that while M 92 is a very metal-poor cluster, comparable to NGC 6397, both M 71 and M 13 are rather metal-rich. In fact more metal rich than $[\mathrm{Fe} / \mathrm{H}]=-1.5$, which is roughly the metal-rich edge of the Spite plateau.

The constancy of Li abundances within the cluster has two important implications:

1. it poses a very stringent upper limit to the maximum intrinsic dispersion allowed for any proposed Li depletion mechanism, which must be less than $0.035 \mathrm{dex}$.

2. the good agreement between the mean $\mathrm{Li}$ abundance in the cluster and that obtained for the field stars suggests that this is in fact the primordial $\mathrm{Li}$ abundance.

The mean value of $A(\mathrm{Li})$ in the cluster, based on 12 stars, including corrections for "standard" depletion and NLTE effects, is $A(\mathrm{Li})=2.34$ with a standard deviation of 0.056 . This is a statistical error to which we must add the systematic error corresponding to $80 \mathrm{~K}$ in the temperature scale, which amounts to $0.06 \mathrm{dex}$ in $\mathrm{Li}$ abundance. Taking this value as the estimate of the primordial $\mathrm{Li}$ abundance, we obtain two possible values for the baryon-to-photon ratio: $n_{\mathrm{b}} / n_{\gamma}=4.3 \times 10^{-10}$ which, using the relation $\Omega_{\mathrm{b}} h^{2}=3.65 \times 10^{-3} \times \eta \times 10^{-10}$, implies $\Omega_{\mathrm{b}} h^{2}=0.016 \pm 0.004$, in good agreement with recent results from the abundance of D (D'Odorico et al. 2001; Levshakov et al. 2002) and ${ }^{3} \mathrm{He}$ (Bania et al. 2002) and from the CMB fluctuation spectrum (de Bernardis et al. 2002); or $n_{\mathrm{b}} / n_{\gamma}=1.5 \times 10^{-10}$ which implies a low baryon density $\Omega_{\mathrm{b}} h^{2}=0.005_{-0.0006}^{+0.0026}$. In spite of the considerable error on the $\mathrm{Li}$ abundance, the error on $\Omega_{\mathrm{b}}$ is dominated by the error on the theoretical predictions of nucleosynthesis.

The lithium content of NGC 6397 supports the primordial origin of lithium in metal-poor stars and the standard big-bang model without need for any new physics.

Acknowledgements. Part of this work was done while PB was at the Observatoire de Paris-Meudon as a visitor. R. Cayrel and M. Spite 
are warmly thanked for many helpful discussions. This research was done with support from the italian MURST/MIUR COFIN2000 grants for the projects "Osservabili stellari di interesse cosmologico" (P.I. V. Castellani) and "Spettroscopia e studio dei processi fisici del Sole e delle stelle di tipo solare" (P. I. G. Peres).

\section{References}

Alonso, A., Arribas, S., \& Martinez-Roger, C. 1996, A\&A, 313, 873

Anthony-Twarog, B. J., \& Twarog, B. A. 2000, AJ, 120, 3111

Bania, T. M., Rood, R. T., \& Balser, D. S. 2002, Nature, 415, 54

Boesgaard, A. M., \& King, J. R. 2002, ApJ, 565, 587

Boesgaard, A. M., \& Tripicco, M. J. 1987, ApJ, 313, 389

Boesgaard, A. M., Deliyannis, C. P., Stephens, A., \& King, J. R. 1998, ApJ, 493, 206

Boesgaard, A. M., Stephens, A., King, J. R., \& Deliyannis, C. P. 2000, Proc. SPIE, 4005, 274

Beveridge, C. R., \& Sneden, C. 1994, AJ, 108, 285

Bonifacio, P., \& Molaro, P. 1997, MNRAS, 285, 847

Bonifacio, P. 2001, in The link between stars and cosmology, ed. M. Chavez, \& D. Mayya (Dordrecht: Kluwer), in press

Cameron, A. G., \& Fowler, W. A. 1971, ApJ, 164, 111

Carlsson, et al. 1994, A\&A, 288, 860

Castellani, V., Ciacio, F., degl'Innocenti, S., \& Fiorentini, G. 1997, A\&A, 322, 801

Castilho, B. V., Pasquini, L., Allen, D. M., Barbuy, B., \& Molaro, P. 2000, A\&A, 361, 92

Cayrel, R. 1988, in The Impact of Very High $S / N$ Spectroscopy on Stellar Physics, ed. G. Cayrel de Strobel, \& M. Spite (Dordrecht: Kluwer), 345

Charbonnel, C., \& Talon, S. 1999, A\&A, 351, 635

Chieffi, A., Domínguez, I., Limongi, M., \& Straniero, O. 2001, ApJ, 554,1159

de Bernardis, et al. 2002, ApJ, 564, 559

Dekker, H., D’Odorico, S., Kaufer, A., Delabre, B., \& Kotzlowski, H. 2000, Proc. SPIE, 4008, 534

Deliyannis, C. P., \& Pinsonneault, M. H. 1997, ApJ, 488, 836

Deliyannis, C. P., Demarque, P., \& Kawaler, S. D. 1990, ApJ, 73, 21

D’Odorico, S., Dessauges-Zavadsky, M., \& Molaro, P. 2001, A\&A, 368, L21

François, P. 1986, A\&A, 160, 264

Fuhrmann, K., Axer, M., \& Gehren, T. 1994, A\&A, 285, 585

Gratton, R., Sneden, C., Carretta, E., \& Bragaglia, A. 2000, A\&A, 354,169

Gratton, R., et al. 2001, A\&A, 369, 87
Guenther, D. B., Kim, Y. -C., \& Demarque, P. 1996, ApJ, 463, 382

Henry, R. B. C., Edmunds, M. G., \& Köppen, J. 2000, ApJ, 541, 660

James, F. 1998, MINUIT, Reference Manual, Version 94.1, CERN, Geneva, Switzerland

Kurucz, R. L. 1993, CD-ROM 13, 18, Smithsonian Astrophysical Observatory, http://cfaku5.harvard.edu/

Lebreton, Y., Perrin, M. -N., Cayrel, R., Baglin, A., \& Fernandes, J. 1999, A\&A, 350, 587

Levshakov, S. A., Dessauges-Zavadsky, M., D’Odorico, S., \& Molaro, P. 2002, ApJ, 565, 696

Lucatello, S., \& Gratton, R. 2002, A\&A, submitted

Michaud, G., Fontaine, G., \& Beaudet, G. 1984, ApJ, 282, 206

Molaro, P. 1997, From Quantum Fluctuations to Cosmological Structures, ASP Conf. Ser., 126, 103

Molaro, P., \& Pasquini, L. 1994, A\&A, 281, L77

Molaro, P., Bonifacio, P., \& Pasquini, L. 1997, MNRAS, 292, L1

Oke, J. B. 1990, AJ, 99, 1621

Pasquini, L., \& Molaro, P. 1996, A\&A, 307, 761

Pasquini, L., Liu, Q., \& Pallavicini, R. 1994, A\&A, 287, 191

Pasquini, L., Randich, S., \& Pallavicini, R. 1997, A\&A, 325, 535

Pasquini, L., Randich, S., \& Pallavicini, R. 2001, A\&A, 374, 1017

Pinsonneault, M. H., Walker, T. P., Steigman, G., \& Narayanan, V. K. 1999, ApJ, 527, 180

Pinsonneault, M. H., Steigman, G., Walker, T. P., \& Narayanan, V. K. 2001, ApJ, 571, in press [astro-ph/0105439]

Randich, S., Pasquini, L., \& Pallavicini, R. 2000, A\&A, 356, L25

Randich, S., et al. 2002, A\&A, submitted

Romano, D., Matteucci, F., Molaro, P., \& Bonifacio, P. 1999, A\&A, 352,117

Ryan, S. G., Norris, J. E., \& Beers, T. C. 1999, ApJ, 523, 654

Salaris, M., \& Weiss, A. 2001, A\&A, 376, 955

Smith, V. V., Lambert, D. L., \& Nissen, P. E. 1998, ApJ, 506, 405

Spite, M., \& Spite, F. 1982a, Nature, 297, 483

Spite, F., \& Spite, M. 1982b, A\&A, 115, 357

Spite, F., \& Spite, M. 1986, A\&A, 163, 140

Swenson, F. J. 1995, ApJ, 438, L87

Théado, S., \& Vauclair, S. 2001, A\&A, 375, 70

Thévenin, F., Charbonnel, C., de Freitas Pacheco, J. A., et al. 2001, A\&A, 373, 905

Vauclair, S. 1988, ApJ, 335, 971

Vauclair, S. 1999, A\&A, 351, 973

Vauclair, S., \& Charbonnel, C. 1995, A\&A, 295, 715

Vauclair, S., \& Charbonnel, C. 1998, ApJ, 502, 372

Ventura, P., D’Antona, F., Mazzitelli, I., \& Gratton, R. 2001, ApJ, 550, L65 